\title{
Study sponsorship and the nutrition research agenda: analysis of cohort studies examining the association between nutrition and obesity
}

\author{
Alice Fabbri ${ }^{1,2}$, Nicholas Chartres ${ }^{2}$ and Lisa A Bero ${ }^{2, *}$ \\ ${ }^{1}$ Centre of Research in Medical Pharmacology, University of Insubria, Varese, Italy: ${ }^{2}$ Charles Perkins Centre and \\ Faculty of Pharmacy, The University of Sydney, Camperdown, NSW 2006, Australia
}

Submitted 3 May 2017: Final revision received 21 June 2017: Accepted 12 July 2017: First published online 30 August 2017

\begin{abstract}
Objective: To categorize the research topics covered by a sample of cohort studies exploring the association between nutrition and obesity; to describe their funding sources; and to explore the association between funding sources and research topics.

Design: Cross-sectional study.

Subjects: Cohort studies retrieved from MEDLINE and PubMed published between 2010 and 2016.

Results: One hundred and twenty-one studies were included. Funding source and conflicts of interest were disclosed in 95.0 and $90.1 \%$ of the studies, respectively. Food industry sponsorship was disclosed in $8.3 \%$ of the studies. Half of the studies analysed the consumption of a single food or food groups, $18.2 \%$ included an analysis of dietary patterns and $17.4 \%$ focused on specific nutrients. Highly processed foods were considered in $48.8 \%$ of the studies and $27 \cdot 3 \%$ considered dietary behaviours (e.g. eating away from home). No statistically significant differences in research topics were observed between industry- and non-industryfunded studies.

Conclusions: Cohort studies focused on more complex exposures (e.g. food or dietary patterns) rather than single nutrients. No significant differences in the research agenda by funding sources were observed. The analysis was limited by the low proportion of studies with disclosed food industry sponsorship.
\end{abstract}

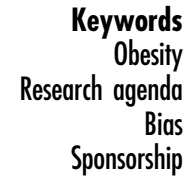

A recent analysis of internal industry documents revealed that decades of research on the link between nutrition and CVD have been shaped by the sugar industry with the aim of deflecting attention away from sucrose as a potential risk factor $^{(1)}$. That new case study bolsters previous concerns about the potential manipulation of the research agenda (namely, the questions being studied) by corporate interests across different fields ${ }^{(2)}$. For example, cigarette companies deflected attention from the hazards of second-hand smoke by funding studies suggesting that other indoor air pollutants were more harmful than tobacco ${ }^{(3)}$.

A systematic review published in 2016 found that few studies have examined potential biases in nutrition research. The twelve studies included in that review focused on the effect of food industry sponsorship on the results, conclusions and methodological quality of nutrition research ${ }^{(4)}$, while potential biases in the nutrition research agenda have not been comprehensively assessed. Apart from case studies based on the analysis of internal sugar industry documents ${ }^{(1,5)}$ and investigative journalism reports that exposed the attempts of a multinational soda company to influence the obesity research agenda $^{(6)}$, a systematic analysis examining whether food industry sponsorship influences the nutrition research agenda has not been done.

We previously conducted a study to explore the association between funding sources and research topics in a sample of randomized controlled trials (RCT) assessing nutrition interventions to address obesity. We found that most of the food industry-sponsored studies involved manipulation of specific nutrients, while less attention was paid to food-level and dietary pattern-level interventions $^{(7)}$. This supports the hypothesis that a reductive focus on nutrients might be strategic for the industry as it can produce results that will allow food companies to market ultra-processed foods using nutrient content claims ${ }^{(8)}$.

However, complex diet-disease relationships cannot always be studied with RCT. Dietary interventions can manipulate only limited components of diet, and are sometimes considered unethical or unfeasible to answer 
questions about the association of diet and diseases ${ }^{(9,10)}$. Therefore, long-term observational studies play a fundamental role in nutrition research and have led to important findings about the complex interrelations between diet and diseases ${ }^{(11)}$. An analysis of 330 papers published in five nutrition journals between January and June 2007 found that observational designs were more frequent than experimental ones $(68.2 v .31 \cdot 8 \%)^{(12)}$. In addition, evidence from observational studies currently informs the development of dietary guidelines ${ }^{(13)}$. Cohort studies are a common and rigorous type of observational study design. Compared with case-control studies, cohort studies can test hypotheses regarding the association between an exposure and multiple outcomes and avoid some of the biases, such as recall bias, that might affect other observational study types ${ }^{(14)}$.

In light of these considerations, the aim of the present study was to evaluate whether the association of research topics and funding sources previously observed in RCT also applies to observational cohort studies. The specific objectives are:

1. to categorize the research topics covered by a sample of cohort studies exploring the association between nutrition and obesity;

2. to describe their funding sources; and

3. to explore the association between funding sources and research topics.

\section{Methods}

\section{Study selection}

We included published cohort studies examining the association between dietary exposures and overweight or obesity. We included studies where the primary or secondary outcomes were BMI score or other measures of overweight and obesity (e.g. waist circumference, fat mass). We included studies published in English, Spanish, French and Italian.

We searched MEDLINE (2010-2016) and PubMed (2010-2016) on 29 July 2016. The search strategy is shown in the online supplementary material, Supplemental File 1. We used a broad search strategy because our analysis was not designed to provide a clinical answer to a particular question (e.g. the association between a specific nutrition exposure and obesity) but to categorize the research topics across a sample of nutrition research studies addressing outcomes relevant to obesity. We restricted our search to studies published from 2010 as international standards on disclosure of funding sources and investigators' conflicts of interest have been mostly developed since that time $\mathrm{e}^{(15,16)}$ and journals' disclosure policies have gradually become more stringent and widespread ${ }^{(17)}$. Thus, as our objective was to explore the association between funding sources and nutrition research topics, we focused on articles that were likely to have funding and conflicts of interest disclosures.

One investigator screened the titles and abstracts for obvious exclusions. When a study could not be rejected with certainty, the decision was made with a second investigator.

\section{Data extraction}

The full text was retrieved for each included study and the following data were collected.

1. Study characteristics: population studied, location, year of publication.

2. Disclosed funding source(s), which were classified as: food industry, pharmaceutical industry, other for-profit entities, governmental agencies, not for profit, mixed funding sources, unknown (when the funding source was disclosed in the article, but information about the sponsor could not be retrieved from the Internet), or no funding disclosed. The food industry category could include transnational food corporations (e.g. Coca-Cola), primary producers (e.g. dairy industry), food processing companies (e.g. meat packing industry), wholesale, distribution and retail companies (e.g. supermarkets, grocery chains), and trade associations (e.g. American Beverage Association).

3. Disclosure of investigators' conflicts of interest as stated in the publications.

4. Research topics as classified below.

The taxonomy we used to code research topics was inspired by a framework for food classification ${ }^{(8)}$ and has already been extensively described elsewhere ${ }^{(7)}$. We coded research topics as follows.

\section{Level of dietary composition}

We assessed whether the study focused on:

- energy level: focus on energy restriction;

- nutrient level: focus on the intake of specific nutrients, micronutrients, compounds (e.g. fat, protein, carbohydrates, vitamin B);

- food level: focus on the consumption of a single food or food groups (e.g. vegetables, fruit, grain foods, meats, fish, eggs, or processed foods such as sugar-sweetened beverages, high-energy snacks, fast foods, etc);

- dietary pattern level: focus on the overall dietary pattern or cuisine (e.g. the Mediterranean diet); or

- not applicable: when the article did not fit in any of the above categories.

To determine the level of dietary composition we extracted the hypothesis of the study. If the hypothesis was not clearly framed, we extracted information on how the authors defined the dietary exposure in the methods and results sections. Each article could fit more than one of the above categories; however, we coded the studies 
according to the most complex level. Each level can therefore include a combination of the lower levels; for example, a study in the food category could include analysis of certain foods (e.g. fruits and vegetables) and studies focusing on a combination of foods and nutrients (e.g. fruits, vegetables and fibre).

Our a priori hypothesis was that the food industry is more likely to fund nutrient-level studies compared with other sponsors to generate evidence in support of its nutritional claims on food products ${ }^{(8)}$.

\section{Level of food processing}

A study was rated as 'yes' if it explored the association between the consumption of highly processed foods, defined as foods that are constructed primarily out of processed-reconstituted and refined-extracted materials (e.g. sugar-sweetened beverages, fried fast foods, breakfast cereals, confectioneries), and obesity-related outcomes. When we pilot-tested the taxonomy, we found that many studies mentioned highly processed foods as one of the many food items that were part of the dietary assessment. Therefore, to be specific rather than sensitive, we decided to code as 'yes' only the studies that mentioned highly processed foods in their primary hypothesis or that reported an adiposity measure related to the consumption of highly processed foods, whether as a single item or as the main components of a dietary pattern. Our a priori hypothesis was that little research focuses on evaluating the effects of the levels of food processing and that the food industry is less likely to sponsor such studies compared with other funding sources ${ }^{(8,18)}$.

\section{Dietary behaviours}

A study was coded as 'yes' if it explored the association between certain dietary behaviours (e.g. meal skipping, eating away from home, eating while watching television, eating dinner together as a family, parental feeding practices) and obesity-related outcomes. Our a priori hypothesis was that the food industry is less likely to fund studies that address dietary behaviours compared with other sponsors.

\section{Double coding}

For the analysis of the nutrition research topics, $30 \%$ of the publications were randomly selected to be coded independently by two reviewers and any disagreement was resolved by consensus. As the percentage of agreement between the two coders was $92.5 \%$, the rest of the sample was single coded.

\section{Analysis}

Categorical variables were described using frequency tables. Cross-tabulations were performed for evaluating possible associations between the funding sources and the research topics using the $\chi^{2}$ test. All analyses were performed using the statistical software package IBM SPSS Statistics version 22.

\section{Results}

\section{Study selection}

As shown in Fig. 1, 1573 studies were identified and 121 met the inclusion criteria. Apart from one retrospective cohort study, all the included studies were prospective cohort studies. They targeted children/adolescents ( $n$ 49; $40.5 \%$ ), adults ( $n 63 ; 52.1 \%$ ) or both ( $n 9 ; 7.4 \%)$. The majority of the studies were conducted in Europe ( $n$ 56; $46.3 \%$ ) and the USA ( $n 31 ; 25.6 \%$ ). The length of observation varied: in fourteen studies it was less than 1 year, in fifty-five studies $1-5$ years, in thirty studies 5-10 years and in twenty-two studies more than 10 years. Five studies looked specifically at how the interaction between genetic variants and weight is mediated by diet.

\section{Disclosure of funding source and investigators' conflicts of interest}

Of the 121 included studies, 95.0\% ( $n$ 115) disclosed their funding source. Of these, 112 studies disclosed the presence of one or multiple sponsors, while in three cases the authors received no specific funding for conducting the study. As Table 1 shows, 40.5\% ( $n$ 49) of the studies were funded by governmental, intergovernmental agencies or other public bodies, 36.4\% ( $n$ 44) by mixed funding sources without any industry involvement, while food industry sponsorship (alone or with other sponsors) was disclosed in $8.3 \%$ ( $n$ 10) of the studies.

Of the 121 included studies, $90 \cdot 1 \%$ ( $n$ 109) contained a conflicts of interest disclosure. In nine studies (7.4\%) the authors disclosed a conflict of interest with the food industry.

\section{Research topics}

Table 2 shows the research topics explored in the included cohort studies. With regard to the level of dietary composition:

- three studies (2.5\%) focused on energy intake (e.g. 'this study aimed to assess associations between baseline objectively measured activity intensity, dietary energy density (DED) and 4-year change in adiposity');

- twenty-one studies (17.4\%) focused on nutrients (e.g. 'the objective of the present study is to investigate the relationship between the macronutrient composition of the usual diet and weight change after 5 years on average');

- sixty-one studies (50.4\%) analysed the consumption of a single food/food groups or a combination of nutrients, energy and foods (e.g. 'our aims were to determine the effects of total and full- and reduced-fat dairy intake in children at 10 year of age on risk of excess total body fat mass (TBFM) and overweight at age 13 year');

- twenty-two studies (18.2\%) included an analysis of dietary patterns (e.g. 'we assessed the association between the adherence to the Mediterranean dietary pattern (MDP), prospective weight change, and the incidence of overweight or obesity'); and 


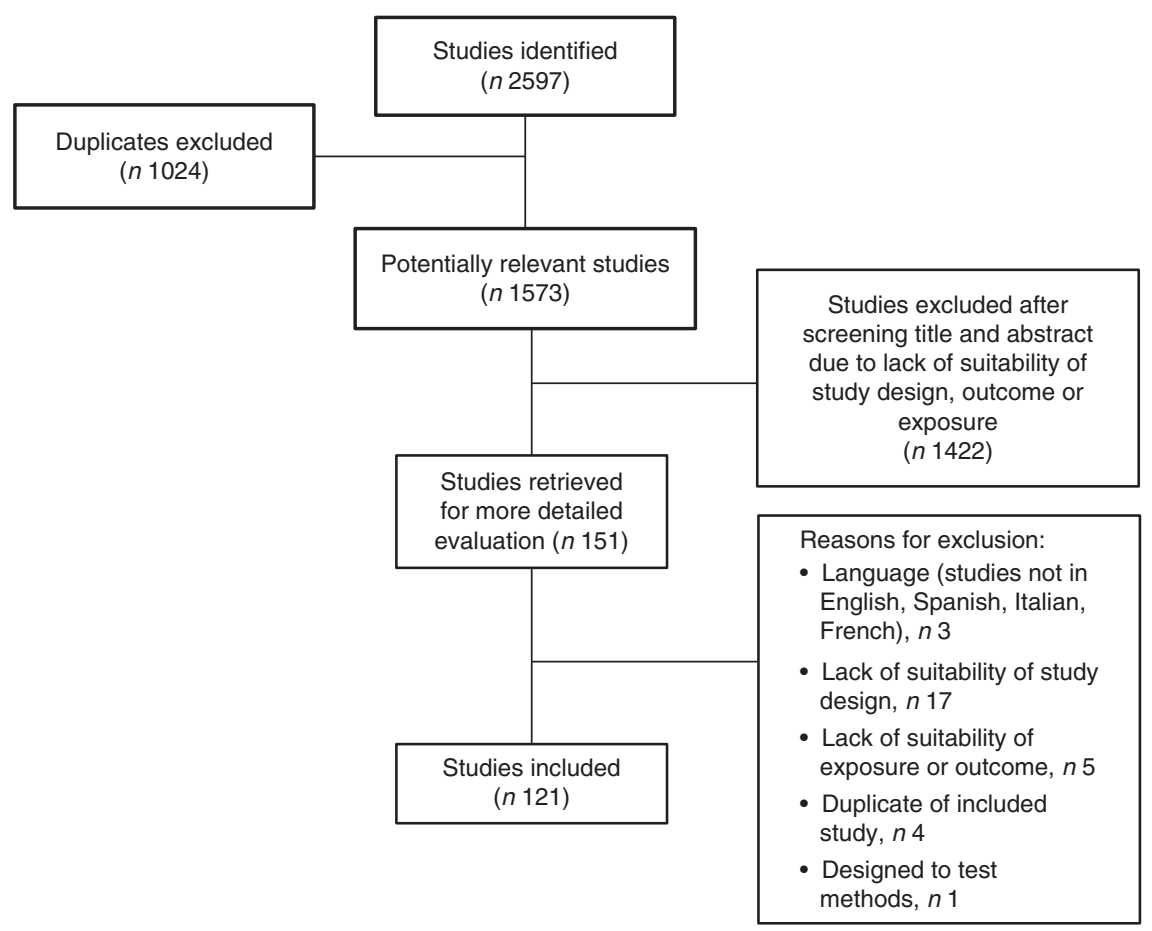

Fig. 1 Flowchart of study selection

Table 1 Categories of funding sources in the sample of cohort studies exploring the association between nutrition and obesity published between 2010 and 2016 ( $n$ 121)

\begin{tabular}{lrr}
\hline Funding source & $n$ & $\%$ \\
\hline Governmental agencies & 49 & 40.5 \\
Mixed funding sources (without industry) & 44 & 36.4 \\
Mixed funding sources (with food industry) & 7 & $5 \cdot 8$ \\
Non-profit & 5 & $4 \cdot 1$ \\
No funding disclosed & 6 & $5 \cdot 0$ \\
Mixed funding sources (with pharmaceutical industry) & 4 & 3.3 \\
No funding for study received & 3 & 2.5 \\
Mixed funding sources with food and pharmaceutical & 2 & 1.7 \\
$\quad$ industries & & \\
Food industry & 1 & 0.8 \\
Total & 121 & $100 \cdot 0$ \\
\end{tabular}

- fourteen studies (11.6\%) did not fit any of the categories related to the level of dietary composition - those fourteen articles addressed dietary behaviours instead (see below).

Highly processed foods were considered in $48.8 \%$ ( $n$ 59) of the studies (e.g. "we examined the association of SCB [sugar-containing beverages] intake at 13 months with BMI development until 6 years and body composition at age 6 years') and $27 \cdot 3 \%$ ( $n$ 33) considered dietary behaviours (e.g. 'we assessed the association between breakfast skipping and BMI among young Chinese children in Hong Kong').

\section{Research topics by funding sources}

The results of the analysis of nutrition research topics by funding sources are presented in Table 3. To test our hypotheses, we compared the research topics of food
Table 2 Nutrition research topics in the sample of cohort studies exploring the association between nutrition and obesity published between 2010 and 2016 ( $n$ 121)

\begin{tabular}{lrr}
\hline & $n$ & $\%$ \\
\hline Level of dietary composition & & \\
$\quad$ Energy & 3 & 2.5 \\
Nutrient & 21 & 17.4 \\
Food & 61 & 50.4 \\
$\quad$ Dietary pattern & 22 & 18.2 \\
$\quad$ Not applicable & 14 & 11.6 \\
Level of food processing & 59 & 48.8 \\
$\quad$ Yes & 62 & 51.2 \\
No & & 27.3 \\
Dietary behaviours & 33 & 72.7 \\
$\quad$ Yes & 88 & \\
No & & \\
\hline
\end{tabular}

${ }^{*}$ For the analysis, we grouped the studies according to the higher level of dietary composition in our taxonomy. Each level can therefore include a combination of the lower levels (e.g. the food category includes studies examining only certain foods and studies focusing on a combination of foods, nutrients and energy).

industry- and non-food industry-sponsored studies. Considering the low proportion of food industry-sponsored studies in our sample, we grouped studies sponsored solely by the food industry ( $n 1)$, studies with mixed food industry and other funding ( $n$ 9), and studies that did not disclose food industry funding but had authors who disclosed a conflict of interest with the food industry or personal support from industry $(n 6)$. The non-food industry category includes studies funded by governmental agencies, nonprofit sector and mixed funding sources without the presence of food industry and the studies that did not receive specific funding ( $n$ 96). We did not include in this analysis the studies funded by pharmaceutical companies 
Table 3 Research topics by funding source for studies that disclosed a sponsor $(n$ 112) in the sample of cohort studies exploring the association between nutrition and obesity published between 2010 and 2016

\begin{tabular}{|c|c|c|c|c|c|}
\hline & \multicolumn{2}{|c|}{$\begin{array}{l}\text { Food industry } \\
\qquad(n 16)\end{array}$} & \multicolumn{2}{|c|}{$\begin{array}{c}\text { Non-food industry } \\
(n 96)\end{array}$} & \multirow[b]{2}{*}{$P$ value } \\
\hline & $n$ & $\%^{*}$ & $n$ & $\% *$ & \\
\hline \multicolumn{6}{|c|}{ Level of dietary composition $†$} \\
\hline Energy & 1 & $6 \cdot 3$ & 1 & 1.0 & 0.050 \\
\hline Nutrient & 6 & 37.5 & 14 & $14 \cdot 6$ & \\
\hline Food & 5 & $31 \cdot 3$ & 50 & $52 \cdot 1$ & \\
\hline Dietary pattern & 1 & $6 \cdot 3$ & 20 & $20 \cdot 8$ & \\
\hline Not applicable & 3 & $18 \cdot 8$ & 11 & 11.5 & \\
\hline \multicolumn{6}{|c|}{ Level of food processing } \\
\hline Yes & 7 & $43 \cdot 8$ & 48 & $50 \cdot 0$ & 0.643 \\
\hline No & 9 & $56 \cdot 3$ & 48 & $50 \cdot 0$ & \\
\hline \multicolumn{6}{|l|}{ Dietary behaviours } \\
\hline Yes & 4 & $25 \cdot 0$ & 26 & $27 \cdot 1$ & 0.862 \\
\hline No & 12 & $75 \cdot 0$ & 70 & $72 \cdot 9$ & \\
\hline
\end{tabular}

${ }^{*}$ Column percentages are calculated.

tFor the analysis, we grouped the studies according to the higher level of dietary composition in our taxonomy. Each level can therefore include a combination of the lower levels (e.g. the food category includes studies examining only certain foods and studies focusing on a combination of foods, nutrients and energy).

and the ones with undisclosed funding sources, therefore the total number of included studies is 112 .

As shown in Table 3, the most represented category in studies sponsored by the food industry was the nutrient level (37.5\%) followed by the food level (31.3\%). Most of the non-food industry-funded studies addressed foods $(52 \cdot 1 \%)$ and $20 \cdot 8 \%$ analysed dietary patterns $\left(\chi^{2}\right.$ test: $P=0.050)$. No statistically significant differences were observed between the two categories of funding source regarding the level of food processing and the dietary behaviours ( $\chi^{2}$ test: $P=0.643$ and $P=0.862$, respectively).

\section{Discussion}

\section{Study sponsorship and the nutrition research agenda}

Our a priori hypothesis, that there would be statistically significant differences in the research topics funded by the food industry compared with other funders, was not confirmed. Our focus on articles published from $2010 \mathrm{did}$ maximize the proportion of studies (95.0\%) with disclosed funding sources. Nevertheless, the analysis of the research topics by funding source was limited by the low proportion of studies with disclosed food industry sponsorship. One explanation could be the possible under-reporting of industry sponsorship or authors' conflicts of interest as has been described in previous studies ${ }^{(19)}$. As the current system for disclosure has flaws, valid methodologies need to be developed to assess the accuracy of disclosed information. Information on conflicts of interests could be sought directly from authors of published studies, but in our experience this is time consuming and yields little additional information. Another alternative is the creation of a centralized, publicly accessible registry for conflicts of interest disclosures that would aggregate information from different sources (e.g. published articles, internal registries of funding agencies) ${ }^{(20)}$. This could be an important source of standardized conflicts of interest information for journal editors, researchers and readers of the scientific literature.

Another reason we did not see a significant association between funding source and research topics could be that we focused on studies examining the relationship between diet and obesity.

Food industry sponsors may not fund nutrition-related research and focus on other topics instead. For example, investigative journalism reports have exposed the attempts of Coca-Cola to influence the obesity research agenda by funding studies that highlighted the role of physical activity in maintaining a healthy body weight, thus deflecting attention from nutrition and particularly from the harmful food commodities that the company markets $^{(6)}$. Clearly one case study cannot be generalized to all food companies, but similar distracting techniques have already extensively been described across other industry sectors $^{(3)}$.

We observed that the most represented category in food industry-sponsored studies was the nutrient level immediately followed by the food level. Most of the nonindustry-funded studies instead addressed foods or dietary patterns. According to some authors, food corporations have strategically exploited the reductive approach to the nutrient composition of diets to market highly processed foods using nutrient content claims, a phenomenon described as 'corporate capture of nutritionism'(8).

The level of food processing was taken into consideration in about half of the cohort studies. Although food processing is a fundamental determinant of overall diet quality and a fundamental driver in the global obesity epidemic $^{(21)}$, it still seems to be an overlooked factor in nutrition science ${ }^{(22)}$. Some researchers have pointed out the risks associated with this approach. For example, unprocessed whole-grain foods and highly processed breakfast cereals could be classified together within the food group of cereals. This has resulted in calls for the adoption of new food classifications based on the extent of industrial processing ${ }^{(22,23)}$. The 2015 Brazilian dietary guidelines are among the few guidelines that explicitly take this issue into account and recommend limiting the intake of processed foods and avoiding those that are ultra-processed $^{(24)}$.

\section{Comparison of the research agenda studied in randomized controlled trials and observational studies}

Our analysis shows that cohort studies focused on examining complex exposures (e.g. foods or dietary patterns) rather than single nutrients or dietary behaviours. 
In addition, highly processed foods were analysed in about half of the studies. In contrast, our previous study on nutrition interventions to address obesity showed that the majority of the included RCT involved manipulations of specific nutrients in a context of reduced energy intake (44.2\%), while there was a gap in the research agenda examining food-level (11.3\%) and dietary pattern-level $(0.9 \%)$ interventions. Highly processed foods were analysed in less than one-third of the interventions and slightly more than half of the RCT addressed dietary behaviours $^{(7)}$.

The gap in the research agenda and particularly the nutritional reductionism that was found in our previous study on RCT was not so evident in the current sample of cohort studies. One explanation for these differences in level of dietary composition studied could be that cohort studies may be more likely than trials to focus on complex exposures in real-word scenarios rather than a single nutrient's manipulation under ideal conditions. Another explanation could be that research topics have changed over time. The cohort analysis included only studies published between 2010 and 2016, while the RCT sample covered the period 1978-2013. The increasing focus on higher levels of dietary composition (e.g. foods and dietary patterns) might be the result of the numerous calls that have been made for an alternative approach to nutrition research, namely a 'top-down' approach that starts from diet patterns and then works backwards to smaller units of analysis (e.g. nutrients) ${ }^{(25,26)}$.

\section{Limitations}

Our study has several limitations. First, we searched only two databases. However, the search strategy was broad and the large number of studies that did not meet our inclusion criteria suggests that the search was sensitive rather than specific. Also, we were not attempting to identify every study relevant to a clinical question but to identify a sample of cohort studies analysing the association between dietary exposures and obesity. Second, we excluded articles published in languages other than English, Spanish, French and Italian. Third, we relied only on the information reported in the publications and did not contact the authors, therefore important aspects of the studies such as details of exposures studied or undisclosed conflicts of interest might have been missed.

\section{Conclusion}

Cohort studies exploring the association between a dietary exposure and obesity outcomes focus on more complex exposures such as foods or dietary patterns rather than single nutrients. No statistically significant differences in the research topics by funding sources were observed; however, the analysis was limited by the low proportion of studies with disclosed food industry sponsorship.
The low proportion of food industry-sponsored studies suggests that a different approach might be needed to explore corporate interests' influence on the research agenda. To characterize the research agenda of the food industry, one option could be to analyse the research projects that food companies disclose on their websites or in annual reports. Another option could be to focus on a different research area as previous investigations have shown how some food and beverage companies have funded studies on topics that could distract from the harmful effects of their products ${ }^{(5,6)}$. The involvement of food companies in sponsoring research on non-nutritionrelated topics could therefore be another interesting area for future research. Lastly, identification of undisclosed conflicts of interest in nutrition research would support analyses of differences between industry- and nonindustry-supported research.

\section{Acknowledgements}

Financial support: This research received no specific grant from any funding agency in the public, commercial or notfor-profit sectors. N.C. has received a scholarship (James Milner PhD scholarship) from the University of Sydney. A.F. has received a scholarship from the University of Insubria (Varese, Italy). Conflict of interest: None. Authorship: A.F., N.C. and L.A.B. conceived the study and designed the data collection tool. A.F. and N.C. collected the data. A.F., N.C. and L.A.B. analysed the data. A.F. wrote the first draft of the paper. N.C. and L.A.B. contributed to the writing of the paper and approved the final version. Ethics of human subject participation: Not applicable.

\section{Supplementary material}

To view supplementary material for this article, please visit https://doi.org/10.1017/S1368980017002178

\section{References}

1. Kearns CE, Schmidt L \& Glantz SA (2016) Sugar industry and coronary heart disease research a historical analysis of internal industry documents. JAMA Intern Med 176, 1680-1685.

2. Bero LA (2005) Tobacco industry manipulation of research. Public Health Rep 120, 200-208.

3. Barnes DE \& Bero LA (1996) Industry-funded research and conflict of interest: an analysis of research sponsored by the tobacco industry through the Center for Indoor Air Research. J Health Polit Policy Law 21, 515-542.

4. Chartres N, Fabbri A \& Bero LA (2016) Association of industry sponsorship with outcomes of nutrition studies: a systematic review and meta-analysis. JAMA Intern Med 176, 1769-1777.

5. Kearns CE, Glantz SA \& Schmidt LA (2015) Sugar industry influence on the scientific agenda of the National Institute 
of Dental Research's 1971 National Caries Program: a historical analysis of internal documents. PLOS Med 12, e1001798.

6. O'Connor A (2015) Coca-Cola funds scientists who shift blame for obesity away from bad diets. The New York Times, 9 August. https://well.blogs.nytimes.com/2015/08/09/cocacola-funds-scientists-who-shift-blame-for-obesity-away-frombad-diets/?_r=0 (accessed May 2017).

7. Fabbri A, Chartres N \& Scrinis G et al. (2017) Study sponsorship and the nutrition research agenda: analysis of randomized controlled trials included in systematic reviews of nutrition interventions to address obesity. Public Health Nutr 20, 1306-1313.

8. Scrinis G (2013) Nutritionism. New York: Columbia University Press.

9. Brannon PM, Taylor CL \& Coates PM (2014) Use and applications of systematic reviews in public health nutrition. Annu Rev Nutr 34, 401-419.

10. Maki KC, Slavin JL, Rains TM et al. (2014) Limitations of observational evidence: implications for evidence-based dietary recommendations. Adv Nutr 5, 7-15.

11. Patro-Golab B \& Szajewska H (2013) Strengths and weaknesses of observational nutritional studies. World Rev Nutr Diet 108, 11-17.

12. Ortiz-Moncada R, Gonzalez-Zapata L, Ruiz-Cantero MT et al. (2011) Priority issues, study designs and geographical distribution in nutrition journals. Nutr Hosp 26, 784-791.

13. Australian Government, National Health and Research Council, Department of Health and Ageing (2013) Eat for Health. Australian Dietary Guidelines. Providing the scientific evidence for healthier Australian diets. https:// www.nhmrc.gov.au/_files_nhmrc/file/publications/n55_ australian_dietary_guidelines1.pdf (accessed April 2017).

14. Mann CJ (2003) Observational research methods. Research design II: cohort, cross sectional, and case-control studies. Emerg Med J 20, 54-60.

15. Committee on Publication Ethics (2011) Code of Conduct and Best Practice Guidelines for Journal Editors.
https://publicationethics.org/files/Code\%20of\%20Conduct_2. pdf (accessed May 2017).

16. International Committee of Medical Journal Editors (2013) Form for Disclosure of Potential Conflicts of Interest. http:// www.icmje.org/conflicts-of-interest/ (accessed May 2017).

17. Bosch X, Pericas JM, Hernandez C et al. (2013) Financial, nonfinancial and editors' conflicts of interest in high-impact biomedical journals. Eur J Clin Invest 43, 660-667.

18. Monteiro C (2011) The big issue is ultra-processing. There is no such thing as a healthy ultra-processed product. World Nutr 2, 333-349.

19. Ruff K (2015) Scientific journals and conflict of interest disclosure: what progress has been made? Environ Health 14, 45 .

20. Dunn AG, Coiera E, Mandl KD et al. (2016) Conflict of interest disclosure in biomedical research: a review of current practices, biases, and the role of public registries in improving transparency. Res Integr Peer Rev $\mathbf{1}, 1$.

21. Moodie R, Stuckler D, Monteiro C et al. (2013) Profits and pandemics: prevention of harmful effects of tobacco, alcohol, and ultra-processed food and drink industries. Lancet 381, 670-679.

22. Monteiro CA, Levy RB, Claro RM et al. (2010) A new classification of foods based on the extent and purpose of their processing. Cad Saude Publica 26, 2039-2049.

23. Monteiro CA, Cannon G, Moubarac JC et al. (2017) The UN Decade of Nutrition, the NOVA food classification and the trouble with ultra-processing. Public Health Nutr (Epublication ahead of print version).

24. Ministry of Health of Brazil (2015) Dietary Guidelines for the Brazilian Population. http://bvsms.saude.gov.br/bvs/ publicacoes/dietary_guidelines_brazilian_population.pdf (accessed April 2017).

25. Jacobs DR \& Tapsell LC (2013) Food synergy: the key to a healthy diet. Proc Nutr Soc 72, 200-206.

26. Jacobs DR \& Steffen LM (2003) Nutrients, foods, and dietary patterns as exposures in research: a framework for food synergy. Am J Clin Nutr 78, 3 Suppl., 508S-513S. 